\section{Review Article}

Check for updates

\title{
CMR Parametric Mapping as a Tool for Myocardial Tissue Characterization
}

\author{
Vanessa M. Ferreira (D, SB, MD, DPhil, and Stefan K. Piechnik (D, MScEE, PhD, DSc \\ Oxford Centre for Clinical Magnetic Resonance Research, Division of Cardiovascular Medicine, Radcliffe \\ Department of Medicine, University of Oxford, Oxford, United Kingdom
}

Received: Apr 15, 2020

Accepted: Apr 23, 2020

Correspondence to

Vanessa M. Ferreira, SB, MD, DPhil

Oxford Centre for Clinical Magnetic Resonance

Research, Division of Cardiovascular Medicine,

Radcliffe Department of Medicine, University

of Oxford, John Radcliffe Hospital, Level O -

OCMR, Headley Way, Oxford, OX3 9DU, United Kingdom.

E-mail: vanessa.ferreira@cardiov.ox.ac.uk

Copyright $($ ) 2020. The Korean Society of Cardiology

This is an Open Access article distributed under the terms of the Creative Commons Attribution Non-Commercial License (https:// creativecommons.org/licenses/by-nc/4.0) which permits unrestricted noncommercial use, distribution, and reproduction in any medium, provided the original work is properly cited.

ORCID iDs

Vanessa M. Ferreira (ID

https://orcid.org/0000-0002-0046-7634

Stefan K. Piechnik (D)

https://orcid.org/0000-0002-0268-5221

Funding

Drs. Ferreira and Piechnik acknowledge support from the National Institute of Health Research (NIHR) Biomedical Research Centre, Oxford, and the British Heart Foundation Centre of Research Excellence, Oxford. Dr. Ferreira is funded by the British Heart Foundation.

\begin{abstract}
Cardiovascular magnetic resonance (CMR) is the current gold standard for imaging cardiac anatomy, function, and advanced myocardial tissue characterization. After cine, late gadolinium enhancement (LGE), and perfusion imaging, parametric mapping is widely regarded as the 4th era of myocardial CMR development. In contrast to conventional CMR tissue characterization techniques, which rely on relative variations in image intensities to highlight abnormal tissues, parametric mapping provides direct visualization of tissue MR properties such as $\mathrm{T} 1, \mathrm{~T} 2$ and $\mathrm{T} 2{ }^{\star}$ in absolute denominations (e.g. in milliseconds). Presentation as pixel-wise parametric maps adds spatial information for a more complete assessment of the myocardium. Advantages of parametric mapping include direct, quantitative comparisons inter- and within-individuals, as well as detection of diffuse disease not evident on conventional CMR imaging, without the need for contrast agents. CMR parametric mapping methods have matured over the past decade into clinical tools, demonstrating not only clinical utility but added value in a wide range of cardiac diseases. They are particularly useful for the evaluation of acute myocardial injury, suspected infiltration and heart failure of unclear etiology. This review discusses the background of parametric mapping, particularly T1-, T2- and ECV-mapping, general magnetic resonance physics principles, clinical applications (including imaging protocols, image analysis and reporting guidelines), current challenges and future directions. CMR parametric mapping is increasingly available on routine clinical scanners, and promises to deliver advanced myocardial tissue characterization beyond conventional CMR techniques, ultimately helping clinicians to benefit patients in their clinical management.
\end{abstract}

Keywords: Cardiovascular magnetic resonance; Parametric mapping; T1-mapping; T2-mapping; Myocardial tissue characterization

\section{INTRODUCTION}

Cardiovascular magnetic resonance (CMR) is the current non-invasive imaging gold standard for assessing cardiac anatomy, function and, importantly, myocardial tissue characterization. Over the past 2 decades, CMR technology had advanced in important developmental stages in clinical applications. 


\section{Conflict of Interest}

Drs. Ferreira and Piechnik acknowledge support from the National Institute of Health Research (NIHR) Biomedical Research Centre, Oxford, and the British Heart Foundation Centre of Research Excellence, Oxford. Dr. Ferreira is funded by the British Heart Foundation. Dr. Piechnik has patent authorship rights for U.S. patent 9285446 B2 (systems and methods for shortened modified Look-Locker inversion recovery [ShMOLLI] cardiac gated mapping of T1), granted March 15, 2016; all rights transferred to Siemens Medical. Patents pending: Zhang; Piechnik; Ferreira et al. "A method for identity validation and quality assurance of quantitative magnetic resonance imaging protocols". (Oxford University Innovation project \#16435); Hann; Piechnik; Ferreira et al. "Method and Apparatus for Quality Prediction" (Oxford University Innovation project \#16045).

Author Contributions

Conceptualization: Ferreira VM; Writing original draft: Ferreira VM; Writing - review \& editing: Ferreira VM, Piechnik SK.
Firstly, CMR cine imaging can accurately determine cardiac anatomy, allowing quantification of chamber volumes, cardiac mass and function. Secondly, late gadolinium enhancement (LGE) imaging allowed the non-invasive visualization of areas affected by myocardial fibrosis, conferring clinicians the unique ability to differentiate ischemic from non-ischemic pathologies based on typical LGE patterns. Thirdly, CMR perfusion imaging allowed the assessment of impaired myocardial perfusion in high spatial resolution as a means of detecting significant coronary artery disease (CAD).

CMR parametric mapping is widely regarded as the 4th era of myocardial CMR development, which include T1-, T2- and extracellular volume (ECV)-mapping. The European Society of Cardiology has named T1 and T2-mapping as one of six most innovative imaging methods in the evaluation of heart failure patients, ${ }^{1)}$ and the most recent expert consensus statement on CMR in Nonischemic Myocardial Inflammation (2018) had updated the Lake Louise Criteria to include parametric mapping as diagnostic criteria for myocarditis. ${ }^{2)}$

This review will focus on the principles and clinical applications of T1-, T2- and ECV-mapping, including challenges and future directions.

\section{PARAMETRIC MAPPING}

Parametric magnetic resonance (MR) relaxometry mapping methods (such as T1- and T2mapping) are quantitative magnetic resonance imaging (MRI) techniques that provide a pixelby-pixel representation of absolutely denominated numerical T1 or T2 properties, expressed in units of time (e.g. milliseconds). T1 and T2 relaxation times can be used to infer tissue type and composition in view of the surrounding environment. This is in contrast to conventional T1- or T2-weighted MRI methods, which rely on the relative image signal intensities to highlight and label areas deemed abnormal, compared to areas deemed normal. Traditional MRI is subject mainly to visual assessment, but allows semi-quantitative analysis in terms of signal intensity ratios or differences. In this regard, conventional MR images are not ideal to detect diffuse and homogenous disease presentations. The advantages of directly quantitative parametric mapping include that they can detect diffuse disease by comparing to previously established normal ranges. This also allows direct quantitative comparison of maps within individuals longitudinally over time, and between individuals.

It is important to distinguish "mapping" from "relaxometry" or "quantification". T1/T2 relaxometry or quantification refers to the overall numerical value (of $\mathrm{T} 1, \mathrm{~T} 2$ ), obtained from either individual images or maps, without necessarily presenting them in the form of the pixel-by-pixel maps. A good example is the current clinical practice of using $\mathrm{T} 2{ }^{\star}$ relaxometry to detect myocardial iron overload. This uses a series of $\mathrm{T} 2{ }^{\star}$ weighted images with different echo times (TEs), and drawing a region of interest (ROI) in the septum of a mid-ventricular slice, to assess signal decay and produce a single $\mathrm{T} 2{ }^{\star}$ value for that region in a patient (Figure 1) ${ }^{3}$; it does not present the findings in a pixel-by-pixel T2* "map". This is in contrast to the use of a map to display the spatial information (Figure 2). ${ }^{4}$ Mapping is often used for regional quantification, by averaging pre-quantified pixel values - this process is different from relaxometry, and produces different numerical results. So in our previous example, the T2^ relaxometry value (derived from a septal ROI) will be different from the $\mathrm{T} 2^{\star}$ value derived from a septal ROI on a $\mathrm{T} 2 \star$ "map"; this is because the T2^ map already has pre-quantified $\mathrm{T} 2{ }^{\star}$ values for each pixel, and the ROI represents an average of these individual pixels. This is analogous to the difference between average of ratios and ratio of averages. ${ }^{5)}$ 
A
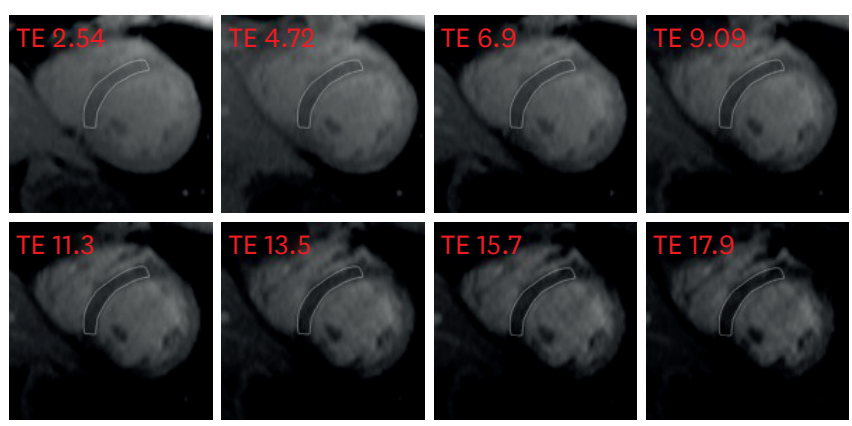

C
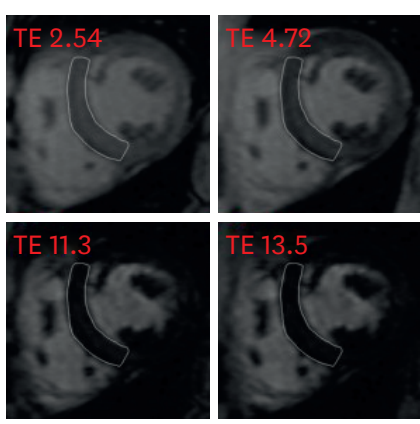
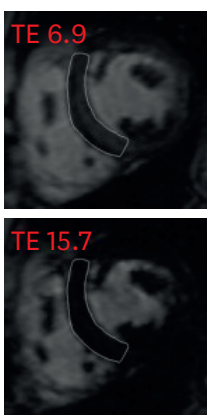
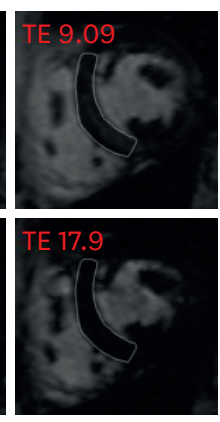

B

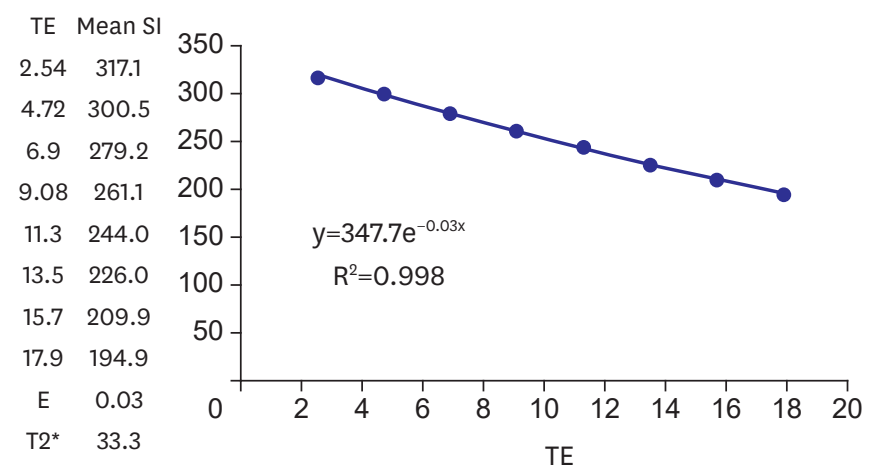

D

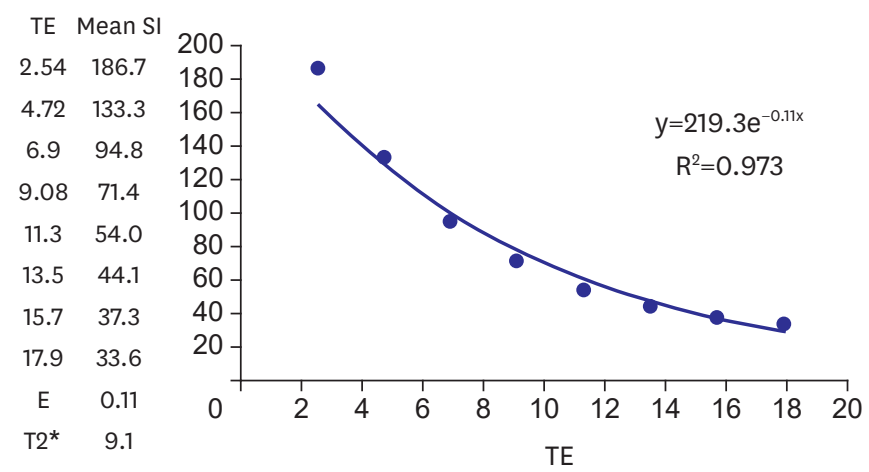

E

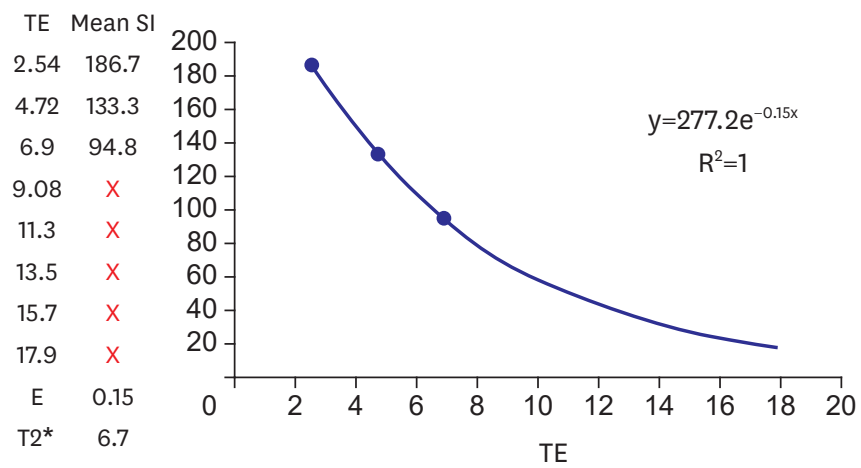

Figure 1. T2* imaging to assess myocardial iron overload. (A) T2* scan of a normal heart showing slow signal loss with increasing TE. (B) Decay curve for normal heart $(\mathrm{T} 2 *=33.3 \mathrm{~ms}$ ). (C) Heavily iron overloaded heart. Note there is substantial signal loss at $\mathrm{TE}=9.09$. (D) Decay curve for heavily iron overloaded heart showing rapid signal loss with increasing TE. The curve plateaus as myocardial signal intensity falls below background noise. (E) Values for higher TEs are removed (truncation method) resulting in a better curve fit and a lower T2* value. As originally published by BioMed Central in Schulz-Menger et al. Journal of Cardiovascular Magnetic Resonance 2013;15:35.3)

$\mathrm{TE}=$ echo time .

\section{T1-MAPPING}

$\mathrm{T} 1$ (or spin-lattice) relaxation time is the characteristic decay constant governing the recovery of longitudinal magnetization $(\mathrm{Mz}$ ) back towards its thermal equilibrium after perturbation of nuclear spins by a designated electromagnetic pulse in very simple materials. The T1 time depends on the physical composition of a material sample; in living tissue, this is deemed to depend on tissue type and its physical and biochemical milieu. In reality, living tissue is 


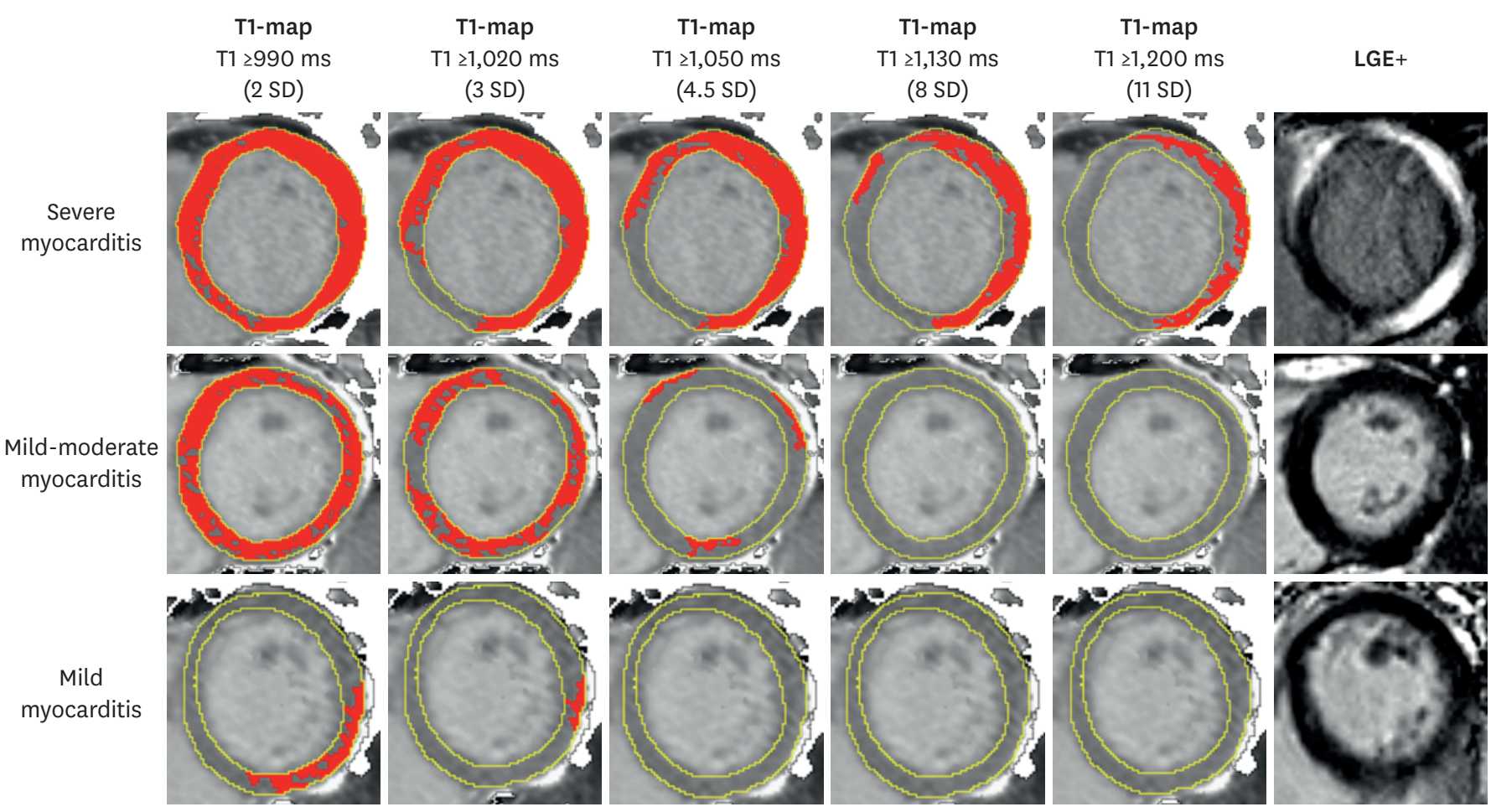

Figure 2. T1-maps using incremental thresholds demonstrate the predominantly non-ischemic pattern of injury across a spectrum of acute myocarditis. Red indicates areas of myocardium with a T1 value above the stated threshold of at least $40 \mathrm{~mm}^{2}$ in contiguous area. T1 threshold of $990 \mathrm{~ms}$ was previously validated for the detection of acute myocardial edema; other thresholds were selected for illustrative purposes. As originally published by Biomed Central in Ferreira et al. Journal of Cardiovascular Magnetic Resonance 2014;16:36. ${ }^{4)}$

$\mathrm{LGE}=$ late gadolinium enhancement; $\mathrm{SD}=$ standard deviation.

a complex, compartmentalized system. In vivo, the Mz recovery depends on magnetization exchange between visible and invisible tissue compartments, $\mathrm{T} 2$ and characteristic tissue resonance frequencies, so different types of MR experiments show different recovery profiles. ${ }^{67)}$ Thus, the Mz recovery is more complex than can be described by a single constant, which makes using a single $\mathrm{T} 1$ measurement a bold approximation in vivo.

MR sequences for cardiac T1-mapping are wide ranging, with the most common variants based on inversion-recovery, saturation-recovery or their hybrid combinations. ${ }^{8}$ Inversionrecovery based T1-mapping techniques are the most commonly-used, since the modified Look-Locker inversion recovery (MOLLI) method was first proposed in 20049) for cardiac T1-mapping (Figure 3). ${ }^{10)}$ MOLLI-based sequences have many variants, starting from the shortened MOLLI (ShMOLLI 2009, Figure 3), ${ }^{10)}$ which was designed to measure short and long T1s within a single sequence. Subsequently, many MOLLI variants have emerged, with different sampling schemes. Some are optimized for measuring selected short or long T1 and selected heart rate ranges, where the radiographer is required to select the most appropriate method at the time of scanning.

Each T1-mapping method has its own metrological properties, and may have its own normal range different from any other T1 mapping method. This is because in vivo T1 values of any organ depend on a vast number of factors that can affect their measurement, from technical (such as the choice of sequence as explained above, its parameters, software version), MR hardware (such as field strength, gradients, etc), and physiologic factors (such as sex, age, heart rate, and temperature). 


\section{A MOLLI sequence}

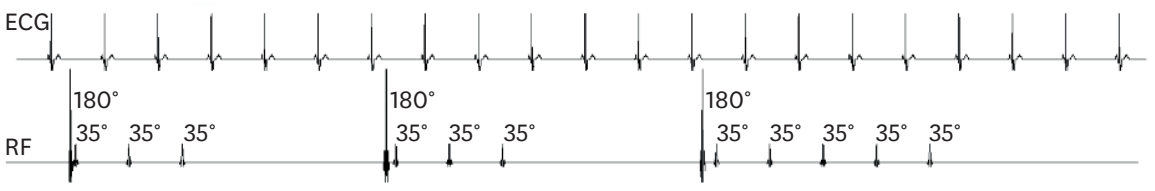

$\mathrm{Mz}(\mathrm{t})[\mathrm{au}]$

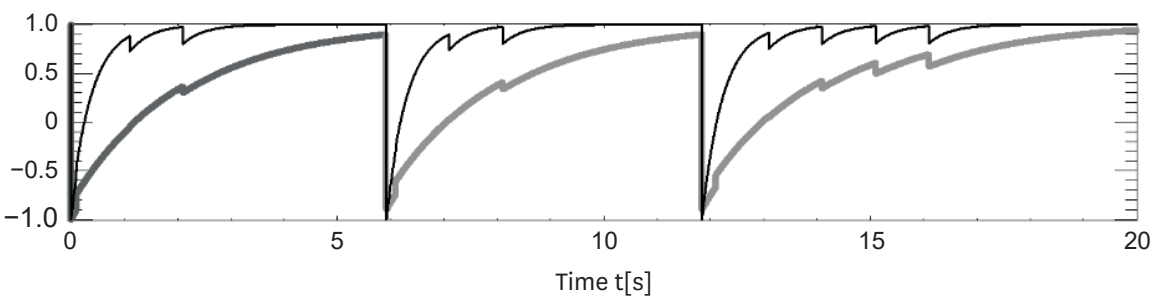

Signal(t) [au]

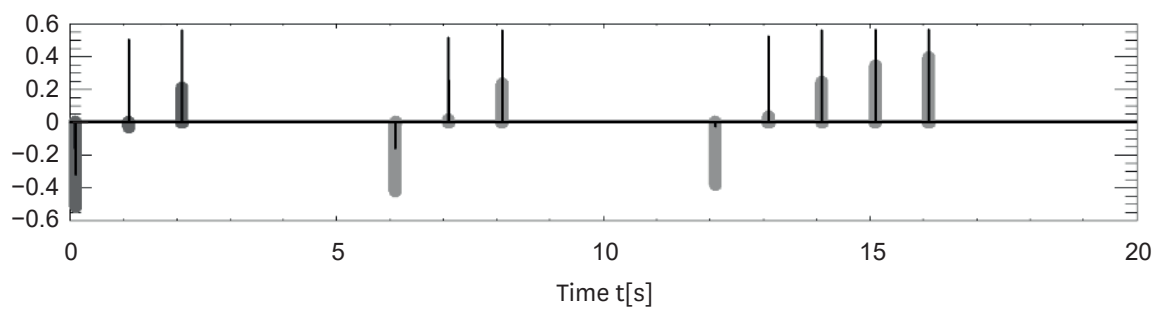

B ShMOLLI

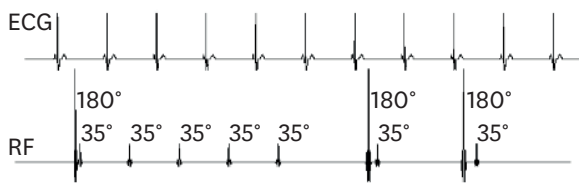

$\mathrm{Mz}(\mathrm{t})[\mathrm{au}]$

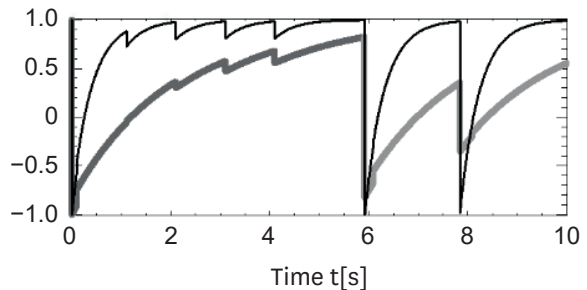

Signal (t) [au]

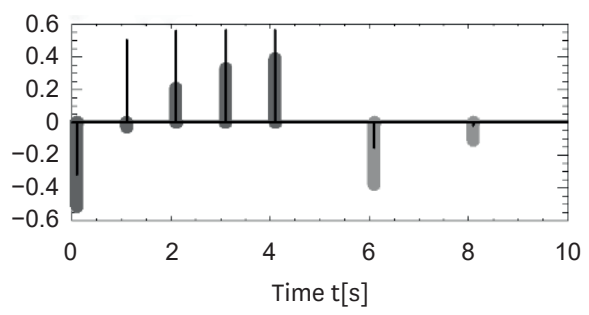

Figure 3. ECG-gated pulse sequence schemes for simulation of (A) MOLLI and (B) ShMOLLI at a heart rate of $60 \mathrm{bpm}$. SSFP readouts are simplified to a single $35^{\circ}$ pulse each, presented at a constant delay time TD from each preceding $R$ wave. The $180^{\circ}$ inversion pulses are shifted depending on the IR number to achieve the desired first TI of 100, 180 and $260 \mathrm{~ms}$ in the consecutive IR experiments. The plots below represent the evolution of longitudinal Mz for short T1 (400 ms, thin lines) and long $\mathrm{T1}$ (2,000 ms, thick lines). Note that long epochs free of signal acquisitions minimise the impact of incomplete Mz recoveries in MOLLI so that all acquired samples can be pooled together for T1 reconstruction. In ShMOLLI the validity of additional signal samples from the 2nd and 3rd IR epochs is determined by progressive nonlinear estimation. As originally published by BioMed Central in Piechnik S et al. Journal of Cardiovascular Magnetic Resonance 2010;12:69. ${ }^{10)}$

$\mathrm{IR}=$ inversion recovery; $\mathrm{MOLLI}=$ modified Look-Locker inversion recovery; $\mathrm{Mz}=$ longitudinal magnetisation; ShMOLLI = shortened modified Look-Locker inversion recovery; SSFP = steady-state free precession; TD = trigger delay.

\section{Native T1-mapping}

Native T1-mapping refers to T1-mapping at rest and before the administration of any contrast or stress agents, including exercise stress. Native T1 represents a composite signal from both the intracellular and extracellular compartments. Each tissue type has a specific normal range of T1 values, deviation from each may indicate disease or change in physiology. Native T1-mapping methods are characterized by a relatively narrow normal range of myocardial $\mathrm{T} 1$ with a small standard deviation, ${ }^{1112}$ and have been demonstrated in multiple studies to be sensitive to changes in a wide range of common myocardial diseases. ${ }^{813)}$ Native T1 values increase with free water content in tissue, and T1-mapping is particularly useful for detecting acute myocardial inflammation/edema, ${ }^{414-16)}$ or in chronic pathologies in which the myocardium has an expanded interstitial space where free water can accumulate, such as in areas of chronic fibrosis or infiltration of the ECV by amyloidosis. ${ }^{817)}$ Native T1 values are lowered by iron or fat content within the myocardium, and are also useful to detect myocardial siderosis and Anderson-Fabry disease. ${ }^{18-21)}$

The biggest advantage of native T1-mapping is myocardial tissue characterization without the need for contrast agents. It readily detects changes in acute myocardial infarction, ${ }^{10222-25)}$ acute myocarditis, ${ }^{415)}$ and cardiac amyloidosis ${ }^{1726-28)}$ - these typically lead to very high native T1 values (Figures 4 and 5). ${ }^{1729)}$ In cardiomyopathies, T1-mapping have been shown to detect abnormalities not detectable by LGE, such as in dilated cardiomyopathy (DCM) and hypertrophic cardiomyopathy (HCM), in which "apparently-normal" myocardial segments with normal wall thickness and without 
Native T1-map
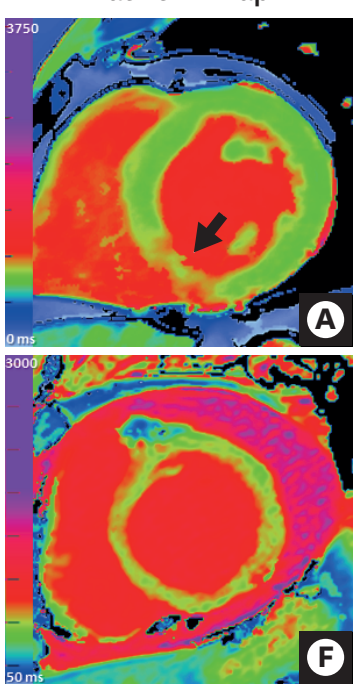

T2-map
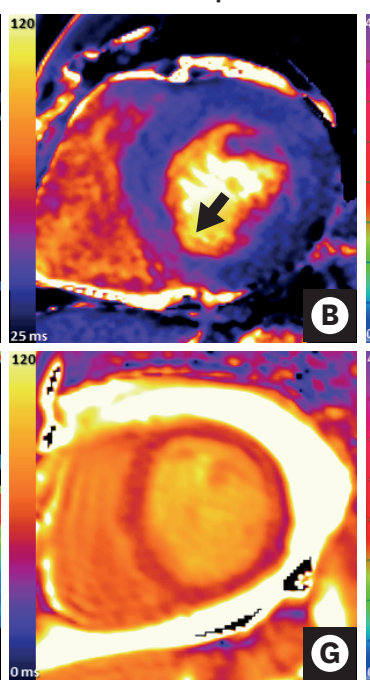

Post-Gd T1-map
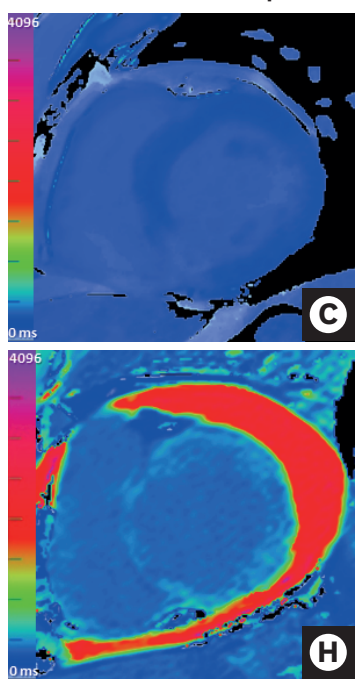

ECV map
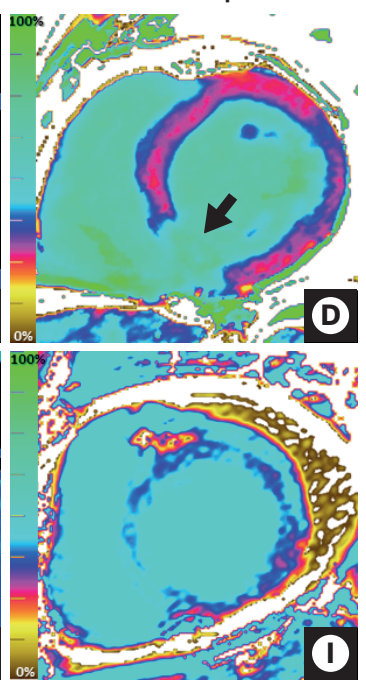

LGE

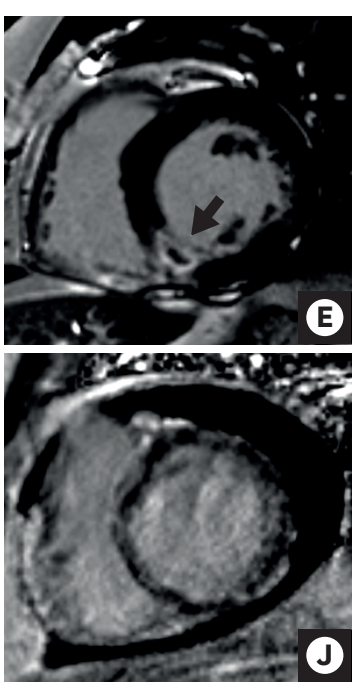

Figure 4. CMR mapping and LGE imaging in acute myocardial pathologies. (Top row) CMR (3 Tesla) images of a patient with an acute myocardial infarction in the inferior septum. (A) Native T1-map (ShMOLLI) showing significantly increased T1 values in the inferior septum (1,407 ms vs. 1,159 ms in remote myocardium in the anterior wall; normal ShMOLLI T1 at 3T =1,166 $\pm 60 \mathrm{~ms}$ ). (B) T2-map showing increased T2 values in the inferior septum (arrow, 55 ms vs. 39 ms in remote myocardium in the anterior wall). (C) Post-gadolinium contrast T1-map. (D) ECV map showing significantly increased ECV in the inferior septum (59\% vs. 30\% in remote myocardium in the anterior wall). (E) LGE imaging showing transmural enhancement with a core area of microvascular obstruction in the inferior septum (arrow). (Bottom row) CMR (1.5 Tesla) images of a patient with acute myocarditis. (F) Native T1-mapping using the ShMOLLI method showed significantly increased global myocardial T1 values (1,060 ms; normal ShMOLLI T1 at 1.5T = 962 $\pm 25 \mathrm{~ms}$ ). (G) T2-map showed increased global myocardial T2 values (59 ms), consistent with edema. (H) Post-gadolinium contrast T1-map. (I) ECV mapping showed increased global ECV of 36\% (normal 27 $\pm 3 \%$ ). (J) LGE imaging showed small areas of patchy enhancement in a non-coronary distribution.

CMR = cardiovascular magnetic resonance; ECV = extracellular volume; LGE = late gadolinium enhancement; ShMOLLI = shortened modified Look-Locker inversion recovery.

LGE also demonstrate abnormally increased T1 values (Figure 6). ${ }^{830)}$ Native T1-mapping appears to be more sensitive to myocardial iron content than conventional $\mathrm{T}^{\star}{ }^{\star}$ relaxometry, demonstrating low $\mathrm{T} 1$ in cases with normal T2 ${ }^{\star}$ values, ${ }^{18)}$ although how this may affect clinical decision-making awaits further research. Native T1-mapping can also differentiate Anderson-Fabry disease (characterized by genetic alpha-galactosidase deficiency and accumulation of sphingolipids within the myocardium) from other phenotypes of left ventricular hypertrophy (LVH), most notably HCM. In Anderson -Fabry disease, T1-mapping also adds value to conventional to LGE imaging, able to demonstrate different populations of abnormal myocardial areas with low T1, but also high T1 (typically in the basal inferolateral wall characterized by a broad midwall band of LGE). ${ }^{1921)}$ These examples exemplify that T1-mapping adds incremental value to conventional CMR methods like cine and LGE in the assessment of cardiac diseases.

\section{Post-contrast T1-mapping and ECV}

Myocardial T1 is relatively long, and is potently shortened by administration of modest amounts of extravascular gadolinium-based contrast agent (GBCA). The post-contrast T1 measurement by itself (or isolated post-contrast T1) changes dynamically with time as GBCA is cleared from the body, and is affected by many other factors (such as renal function, age, hematocrit) to serve as a reliable biomarker; the preferred outputs are native T1 and ECV. ${ }^{31}$

There is significant interest in the non-invasive assessment of the ECV in various cardiac conditions, especially as a surrogate marker of diffuse myocardial fibrosis (DMF), as this may provide insights into the pathophysiology of a condition, and may even be a novel therapeutic target, such as in non-ischemic cardiomyopathy and heart failure. 


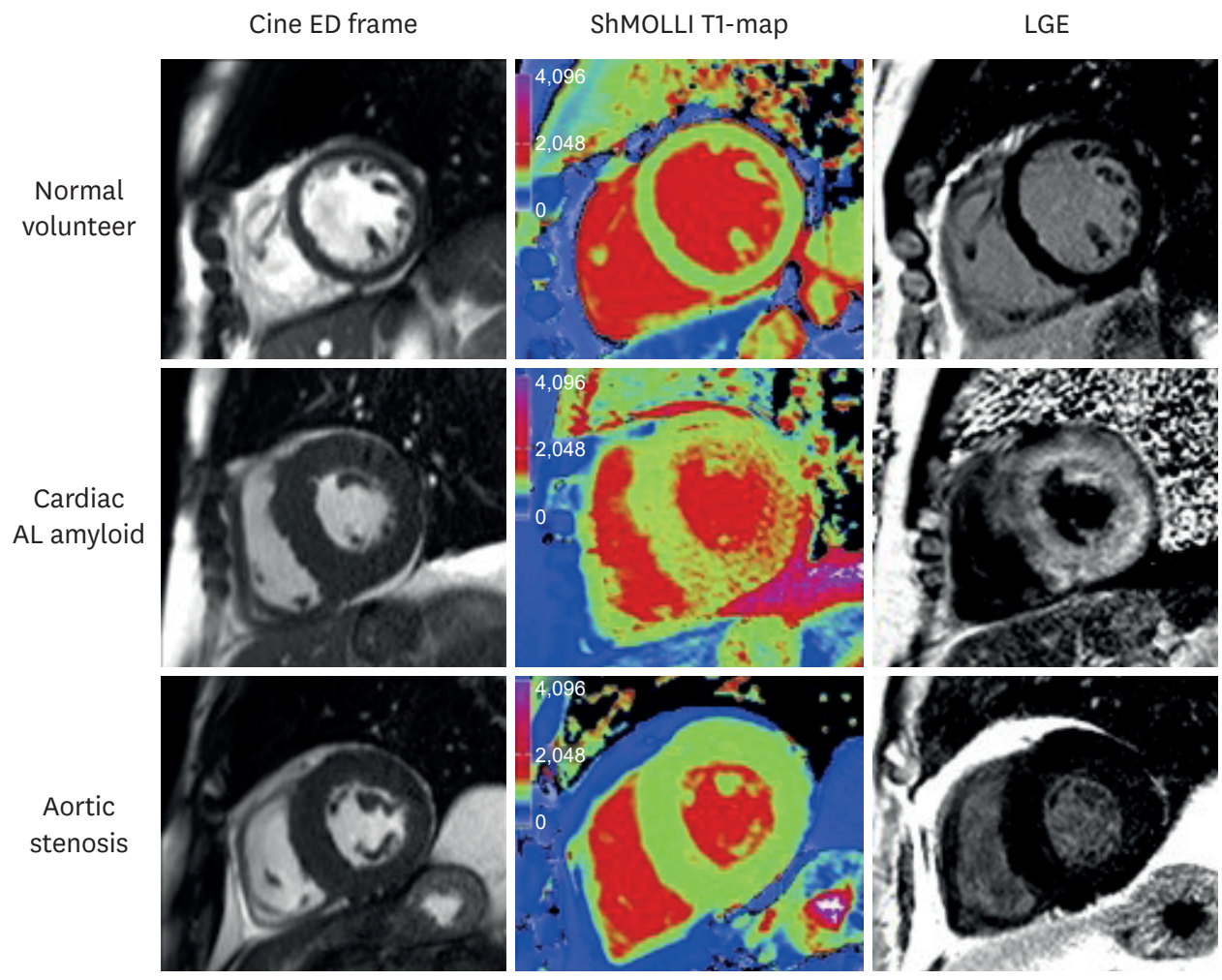

Figure 5. Characteristic examples from CMR scans. CMR end-diastolic frame from cine (left panel), ShMOLLI non-contrast T1 map (middle panel), and LGE images (right panel) in normal volunteer, aortic stenosis patient, and cardiac amyloid patient. Note the markedly elevated myocardial $\mathrm{T} 1$ time in the cardiac amyloid patient (1,170 $\mathrm{ms}$, into the red range of the color scale) compared to the normal control (955 ms) and the patient with aortic stenosis and left ventricular hypertrophy $(998 \mathrm{~ms})$. As originally published in Karamitsos et al. JACC: Cardiovasc Imaging 2013;6:488-97. ${ }^{77}$

$\mathrm{AL}=$ amyloid light chain; $\mathrm{CMR}=$ cardiovascular magnetic resonance; $\mathrm{ED}=$ end diastolic; $\mathrm{LGE}=$ late gadolinium enhancement; ShMOLLI = shortened modified Look-Locker inversion recovery.

The theory of how to measure ECV using CMR is reviewed in detail elsewhere. ${ }^{32}$ Briefly, the myocardial ECV is estimated by measuring the pre- and post-contrast relaxivity changes (R1=1/T1) of myocardium and blood, adjusting the ratio by the known extracellular volume of blood (i.e. 1-Hematocrit). ECV of the myocardium is calculated as follows:

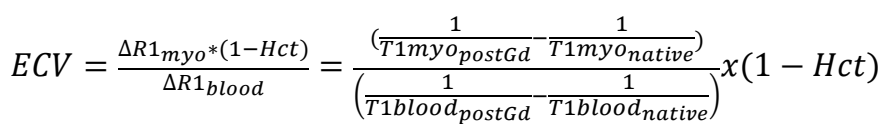

Like native T1, the measurement of ECV comes with several caveats. Firstly, ECV is calculated using T1, and thus depends on the chosen T1-mapping method. Most critically, the formula above depends on an equilibrium distribution of GBCA being achieved between the blood and myocardial interstitial compartments. Initially, an equilibrium-CMR (EQ-CMR) technique, based on a prolonged infusion of GBCA in excess of 30 minutes outside the scanner, assured the steady state. ${ }^{32)}$ However, this is very time-consuming and not practical for routine clinical applications.

Since then, it has been found that, instead of using a prolonged infusion of GBCA, a bolus injection of GBCA, followed by much shorter wait time, is adequate for estimating ECV in 

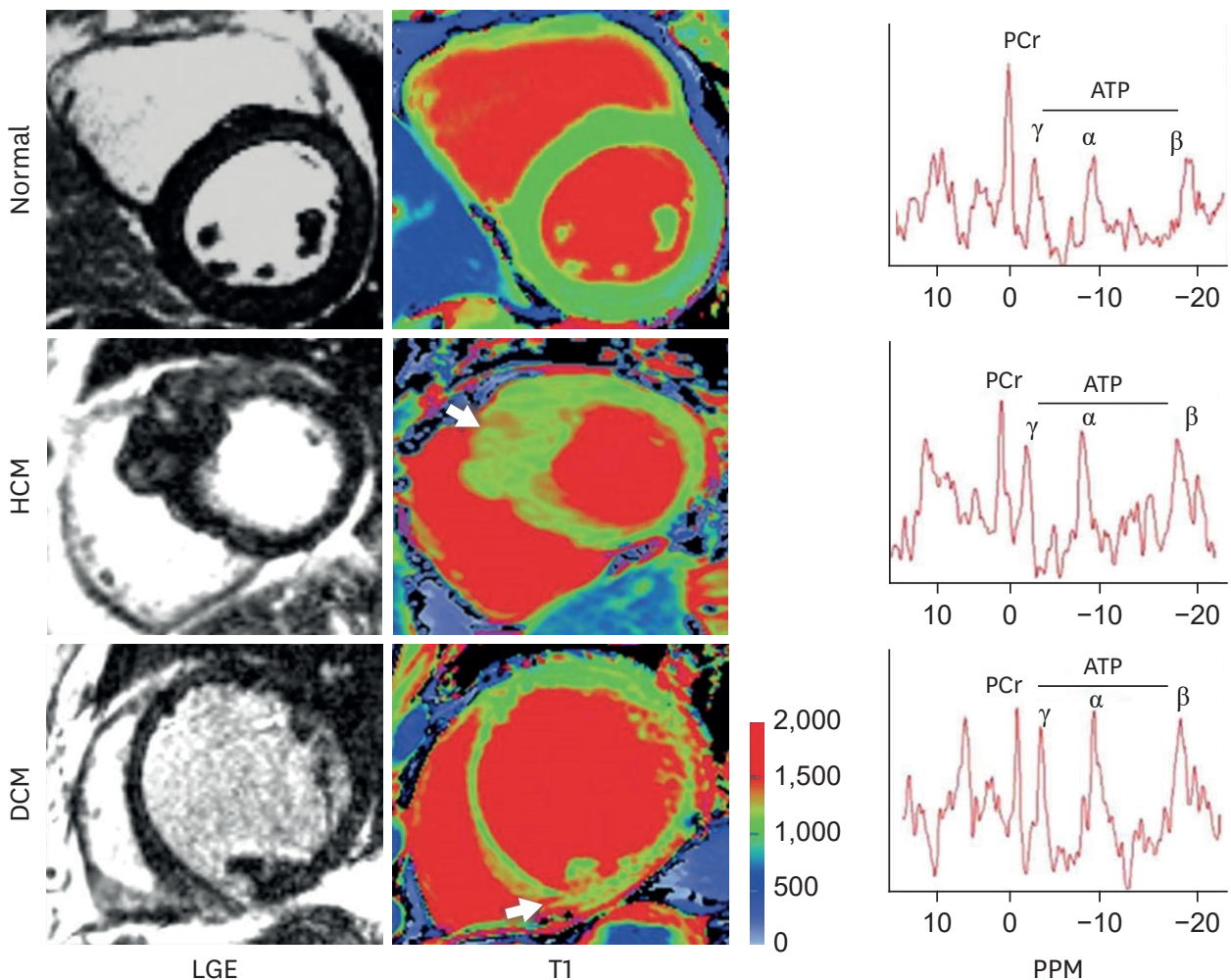

Figure 6. LGE and T1 images in normal, HCM, and DCM. Arrows point to focal areas of LGE and high T1. Note: There is diffusely high $\mathrm{T} 1$ in the septum of HCM and inferior septum/inferior in the DCM. Representative spectra for each condition are shown; PCr indicates phosphocreatine; and ATP adenosine triphosphate. As originally published in Dass S. et al. Circulation Cardiovascular Imaging 2012;5:726-33. ${ }^{30}$ )

ATP = adenosine triphosphate; DCM = dilated cardiomyopathy; HCM = hypertrophic cardiomyopathy; LGE = late gadolinium enhancement; $\mathrm{PCr}=$ creatine phosphate.

most conditions. ${ }^{33)}$ This is called dynamic-equilibrium (DynEq) CMR, which is significantly shorter and more practical. Native T1-mapping is acquired before administration of any GBCA, and post-contrast T1-mapping is acquired after a minimum of 10 minutes post injection of GBCA. However, DynEq-CMR may not be as accurate for very expanded ECVs (e.g. $>40 \%$ ), or conditions characterized by large myocyte volumes or areas with high concentration of GBCA. ${ }^{34)}$

It must be noted that myocardial ECV consists of the interstitial space between myocytes and also within the blood vessels between cells (the intravascular compartment). ${ }^{34)} \mathrm{ECV}$ may be expanded due to diffuse interstitial fibrosis, but also cardiac amyloidosis, edema and expansion of the intravascular compartment by coronary vasodilation, such as in ischemia. ${ }^{8} 35536$ An expanded ECV has been reported to confer poor prognosis especially in patients with heart failure, ${ }^{37 / 38)}$ and cardiac amyloidosis. ${ }^{26)}$ It should be emphasized that an increased ECV is only a surrogate imaging marker of diffuse interstitial fibrosis, and only if confounders (such as edema, amyloidosis, expansion of the intravascular compartment) have been ruled out. ${ }^{8311353) 36(39)}$

\section{Stress T1-mapping}

A novel application of T1-mapping is to acquire T1-maps at rest and during stress conditions (pharmacological or exercise), as a way to assess coronary vasodilatory reserve, which may be impaired in obstructive CAD or non-ischemic pathologies. ${ }^{3533639)}$ The basic principle is that, 
under normal conditions, vasodilatory or exercise stress will cause coronary vasodilation and so an increase in myocardial blood volume, characterized by long T1, to drive up the overall measured T1 in the myocardium. Studies have demonstrated a normal myocardial stress $\mathrm{T} 1$ response to be around $6-7 \%{ }^{35 / 40)}$ In patients with severe aortic stenosis and chronic left ventricular (LV) pressure overload, there exists resting coronary vasodilation even in the absence of obstructive $\mathrm{CAD}$; the coronary vasodilatory reserve is thus diminished, resulting in a blunted stress T1 response. ${ }^{36)}$ Similarly, downstream of a significantly obstructed coronary artery, there will likely be full compensatory resting coronary vasodilation, leading to an elevated resting myocardial T1, which will have little or no capacity to be augmented with further stress, resulting in a significantly abolished stress T1 response. ${ }^{35}$ Initial studies have demonstrated that rest and stress T1-mapping can differentiate between normal myocardium from ischemic and infarcted myocardium according to distinct T1 profiles of these tissue classes (Figure 7). ${ }^{35}$ ) This is a new area of research that may provide a way to assess for ischemia without the need for GBCA, but requires further validation to demonstrate its utility in real-life clinical settings. ${ }^{39)}$

\section{T2-MAPPING}

T2 (or spin-spin) relaxation time is the basic MR constant governing the dephasing of transverse magnetization $(\mathrm{Mx}, \mathrm{y})$ after an MR excitation. There are various sequence designs for T2-mapping, including the use of single-shot balanced steady-state free precession (bSSFP) acquisitions with different T2 prep times, ${ }^{41)}$ gradient and spin echo (GraSE) ${ }^{42}{ }^{43}$ or fast spin echo (FSE)-based pulse sequences. The Society for Cardiovascular Magnetic Resonance (SCMR) Mapping Consensus Statement (2017) recommends T2-prepared bSSFP or gradient echo pulse sequences with a minimum of three differently $\mathrm{T} 2$-weighted images; GraSE or FSE approaches may also be used if there is good published evidence on accuracy and precision suitable for clinical applications. ${ }^{8)}$

A

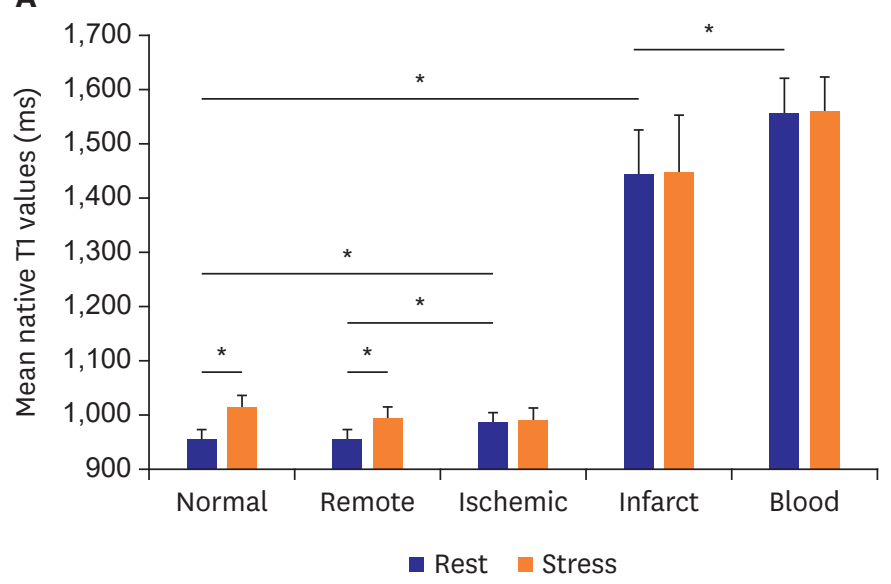

B

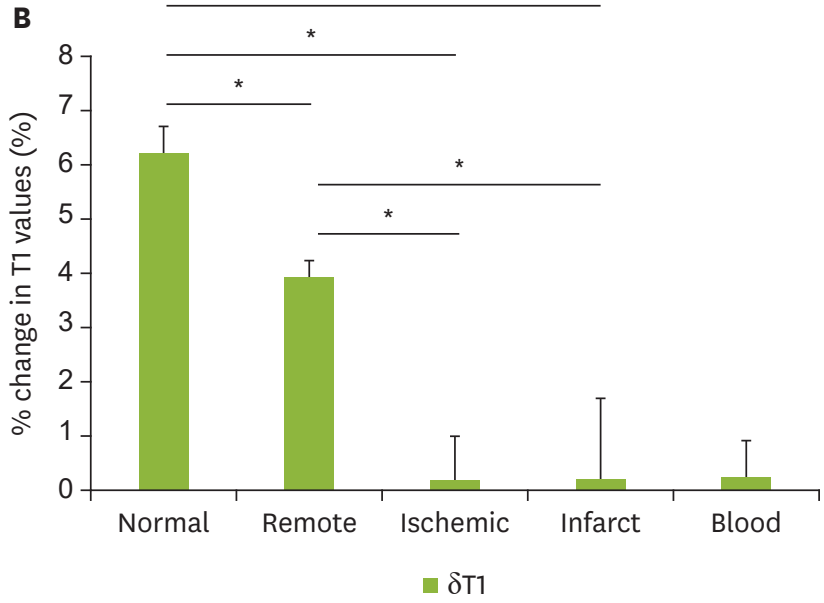

Figure 7. Myocardial T1 at rest and during adenosine stress at 1.5-T. (A) T1 values at rest in normal and remote tissue were similar and significantly lower than in ischemic regions. Infarct T1 was the highest of all myocardial tissue, but lower than the reference left ventricular blood pool of patients. During adenosine stress, normal and remote myocardial $\mathrm{T} 1$ increased significantly from baseline, while $\mathrm{T} 1 \mathrm{in}$ ischemic and infarcted regions remained relatively unchanged. (B) Relative $\mathrm{T} 1$ reactivity ( $\delta \mathrm{T} 1)$ in the patient's remote myocardium was significantly blunted compared to normal, and completely abolished in ischemic and infarcted regions. All data indicate mean \pm 1 SD. Adapted from Liu A. et al. JACC: Cardiovascular Imaging 2016;9:27-36.35) originally published by Elsevier. $\mathrm{SD}=$ standard deviation.

${ }^{*} \mathrm{p}<0.05$. 
Like native $\mathrm{T} 1$, the $\mathrm{T} 2$ time is an MR property of a tissue, affected by the tissue type and its surrounding physical and biochemical milieu. It is a composite signal from both the intracellular and extracellular compartments. The normal range of myocardial T2 values also depends on the choice of the technique, including hardware, software, and physiologic factors, especially sex and age, and most T2-mapping methods exhibit heart rate dependencies. In theory, T2 is also affected by GBCA, but unlike T1, post-contrast myocardial $\mathrm{T} 2$ is too short for these changes to be of clinical use.

$\mathrm{T} 2$ values are sensitive to free water content in tissue, and T2-mapping is considered particularly useful for detecting acute myocardial inflammation/edema. For clinical applications, T2-mapping is commonly used to detect acute myocardial injury or inflammation, such as in acute MI, myocarditis other causes of myocardial infarction with non-obstructive coronary arteries (MINOCA) such as Takotsubo cardiomyopathy, and cardiac transplant rejection. ${ }^{8}$ Compared to conventional T2-weighted CMR imaging, T2mapping obviates some of the limitations, particularly the ability to directly quantify tissue T2 without relying on a reference ROI for comparison as a presumed area of normality-this is particularly relevant in global myocardial edema where there are no unaffected areas within the myocardium, and in systemic inflammatory diseases where even extra-cardiac reference ROIs like skeletal muscle (as needed for dark-blood T2-weighted CMR), may also be inflamed, which can lead to false negatives in detecting myocardial inflammation. ${ }^{215144)}$ The updated Lake Louise Criteria (2018) now includes T2-mapping, in addition to T1-based mapping methods, in the use of CMR for detecting myocardial inflammation. ${ }^{2)}$

\section{IMAGING PROTOCOL}

SCMR periodically publishes updates to recommendations on standardized imaging protocols, and these should be consulted when implementing parametric mapping techniques, in conjunction with the SCMR Mapping Consensus papers. ${ }^{8311}$ Currently, parametric mapping is not standardized and is an evolving field; thus, it is crucial to ensure that any chosen method is implemented correctly, with quality assurance, at a local CMR site, especially before use for diagnosis and clinical applications. Knowledge of and establishment of a local normal range in groups of volunteers is paramount, and where available, the local normal range should be benchmarked against published normal ranges for a chosen mapping method to ensure consistency, both in terms of the mean value and standard deviation. Local manual shimming is especially important for high field strength (3T) ${ }^{8)}$

The following incorporates recommendations from the SCMR Standardized CMR imaging protocols: 2020 update, ${ }^{45}$ SCMR Mapping consensus papers ${ }^{8311}$ and the authors' own expertise:

\section{T1-mapping}

1) Native $T 1$ mapping is performed in the absence of contrast or stress agents, at rest

2) Look Locker imaging (MOLLI or ShMOLLI ${ }^{10)}$ or equivalent) should be used.

3) Diastolic acquisition is best with the exception of atrial fibrillation in which systolic acquisition may be preferred. In patients with higher heart rates, specific sequences designed for these heart rates should be used.

4) Source images should be checked for motion/artefact and imaging repeated if this occurs. If motion correction is used, this needs to be carefully checked for introduction of any new artefacts. ${ }^{46}$ 
5) Slice thickness: $6-8 \mathrm{~mm}$, in-plane resolution $\sim 1.6-2.0 \mathrm{~mm}$

6) The number and orientation of slices obtained will depend upon the indication. At least one short-axis (SA) map should always be obtained.

7) For extracellular volume measurements, T1 mapping should be performed prior to contrast and at least 1 time point between 10 and 30 minutes post contrast bolus. Postcontrast T1-maps should match native T1-maps in slice position and other prescribed imaging parameters (such as field of view and cardiac phase).

8) The hematocrit should be measured just before the scan and, ideally within $24 \mathrm{~h}$ of imaging, for the accurate ECV fraction measurement.

\section{T2-mapping}

1) Multiple alternatives exist, such as T2-prepared single-shot bSSFP sequence acquired with different T2 prep time, GraSE or FSE-based pulse sequences.

2) Slice thickness: $6-8 \mathrm{~mm}$, in plane-resolution $\sim 1.6-2.0 \mathrm{~mm}$

3) Diastolic acquisition is recommended

4) The number and orientation of slices obtained will depend upon the indication. SA maps should always be obtained.

5) Source images should be checked for motion/artefact, including in motion-corrected images, and imaging repeated if this occurs.

\section{Example CMR parametric mapping protocol}

- Localizers

- Pilot scouts

- Cine imaging (horizontal long-axis [HLA], vertical long-axis [VLA], left ventricular outflow tract [LVOT])

- Native T1 mapping

- T2 mapping

$\cdot \mathrm{T} 2{ }^{\star}$ imaging

- Injection of gadolinium-based contrast agents for LGE imaging

- Cine imaging (SA stack)

- LGE (HLA, VLA, LVOT, SA stack)

-Wait at least 10 minutes post-GBCA to acquire post-contrast T1-maps for ECV quantification

\section{IMAGE POST-PROCESSING AND ANALYSIS}

Currently, there is no standardized approach to the image post-processing and analysis of parametric maps, but there are general consensus guidelines based on the SCMR Mapping Consensus statements. ${ }^{831447}$

In general, it is important to ensure that parametric maps are acquired correctly to ensure the best image quality, including T1/T2 curve-fitting. Parametric maps may be processed using commercially-available software packages dedicated for this purpose, or in-house software that has been validated to ensure accurate reporting of T1 and T2 values. Parametric maps may be affected by motion (cardiac, respiratory or body), mistrigerring, susceptibility and many other artefacts. Maps should first be assessed for quality before using for further diagnosis or research purposes, and this may be achieved by examining the T1/T2 curve-fit and/or any accompanying quality control maps. Quality control maps may take the form of maps of R-squared (square of Pearson correlation, i.e. coefficient of explained variance) ${ }^{14)}$ or 

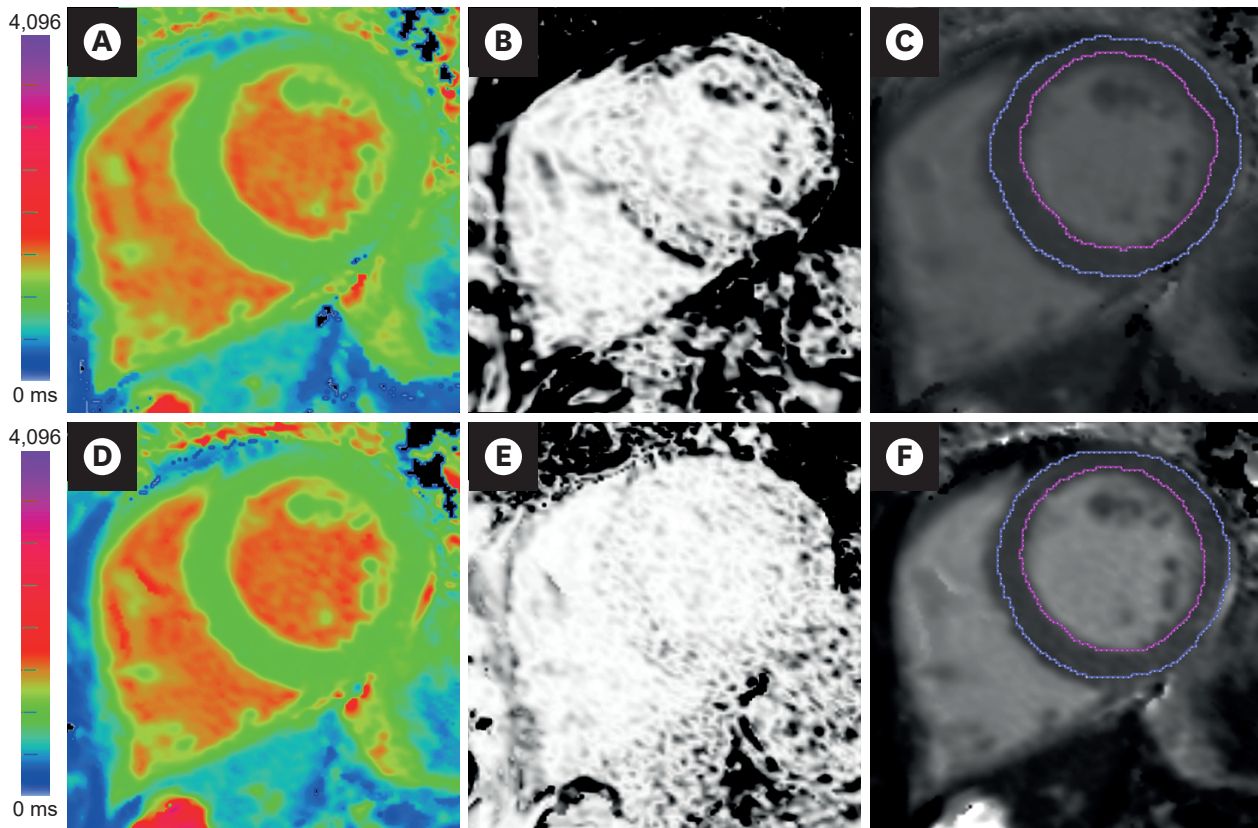

Figure 8. Image analysis of T1-maps. (A) Native T1-map (ShMOLLI at 1.5 Tesla). The acquisition of this T1map suffered from breathing motion, resulting in (B) an $\mathrm{R}^{2}$ map that showed poor $\mathrm{T} 1 \mathrm{fit}$ (black pixels within the LV myocardium) and (C) underestimated average LV myocardial T1 values (T1=928 ms) after myocardial segmentation using epi- and endo-cardial contours (blue and magenta lines). Guided by the suboptimal $\mathrm{R}^{2}$ map, the operator repeated the acquisition with the patient performing a good breath-hold, which produced (D) A good quality native T1-map, as indicated by (E) an $\mathrm{R}^{2}$ map that showed good T1 fit (pixels within the LV myocardium are white), and (F) accurate average LV myocardial T1 values (T1=943 ms). Ensuring good data fit during image acquisition and analysis is paramount in the clinical application of mapping techniques. $\mathrm{LV}=$ left ventricular; ShMOLLI = shortened modified Look-Locker inversion recovery.

estimated T1 standard deviation maps, both of which can provide a visual guide as to whether the parametric map is reliable for clinical diagnosis (Figure 8). ${ }^{29)}$

Once it is determined that the map is of good quality, further image analysis may include visual analysis and more advanced analysis using computer-assisted post-processing. Visual analysis may present the parametric map on a colour scale using look-up tables (LUT) dedicated to highlighting areas of normality versus abnormality, although there is currently no standardized approach for displaying coloured parametric maps. Ideally, the quantitative analysis of parametric maps should employ validated diagnostic thresholds for that mapping method to highlight areas of abnormality (Figure 2), ${ }^{4)}$ but until standardization is achieved, semi-quantitative approaches based on multiples of standard deviations compared to reference ROI or reference cohorts are also used.

For advanced quantitative analysis using dedicated software, ROIs are first placed onto the parametric maps; this may involve placing endo- and epi-cardial contours to segment the LV myocardial ring in SA, which can then be divided into segments (e.g. 6 segments), for global and segmental T1/T2/ECV values. Image analysts should be trained to apply the same standards for image post-processing, ${ }^{48)}$ as the myocardial T1 clearly depends on the partial volume. ${ }^{11)}$ Diagnostic thresholds may then be prescribed to highlight pixels that are above or below a certain T1/T2 value, to give a percent area of abnormality on an image slice (Figure 2).4) Advanced analyses may provide parameters such as measures of tissue heterogeneity, maximum-minimum pixel T1/T2 value differences, or standard deviation (SD) measures 
within an ROI. ${ }^{49}$ Depending on the number of slices of maps available covering the LV, these may be averaged to provide a global LV myocardial T1/T2 value or $\%$ area of abnormality per subject. Separately, manual ROIs may be placed into an area of interest within the myocardium to provide an average T1/T2 value. A septal ROI drawn conservatively only on the mid-ventricular SA slice is sometimes used for diseases expected to be homogenous, but this approach does not maximize the use of these pixel-wise parametric maps, particularly the spatial information and potential regional variations, ${ }^{48}$ to the full extent. Very small ROIs (e.g. $<40 \mathrm{~mm}^{2}$ ) should be avoided.

On the contrary, it is important to note that, for $\mathrm{T} 2{ }^{\star}$ relaxometry, the recommended approach is to use a conservative septal ROI placed in the mid-ventricular SA slice, to avoid any susceptibility artefacts in the lateral wall, and also contamination from the right ventricular (RV) and LV blood pools and epicardial vessels at the superior RV-LV junction that may lead to errors in $\mathrm{T} 2^{\star}$ measurements. To curve-fit the $\mathrm{T} 2^{\star}$ measurements obtained, the truncation method should be used, which excludes T2^ samples acquired at longer TEs to provide accurate $\mathrm{T} 2^{\star}$ estimates (Figure 1$)^{3)}$

For ECV calculation, it is important that the pre-contrast and post-contrast T1-maps are acquired properly and are matching in position and other image parameters (such as field-ofview, cardiac position and phase). ${ }^{50}$ Given the time gap between acquiring pre- and postcontrast T1-maps, ECV quantification cannot be considered as a single acquisition. Endo- and epicardial contours are typically placed to segment the LV myocardium, and an ROI also needs to be placed in the LV blood pool (avoiding papillary muscles and trabeculae) to provide preand post-contrast blood T1 values. Alternatively, contours may be placed in specific regions of interest (e.g. septum or other segments), matching on the pre- and post-contrast T1-maps, to generate region-based ECV estimates. Producing an ECV map is possible to provide spatial information and visualization, subject to successful image co-registration between the pre- and post-contrast T1-maps with potentially differing breath-holds (Figure 9). ${ }^{51)}$ The hematocrit should ideally be drawn from blood on the same day before the CMR scan. If the lab hematocrit is not available, this may be replaced with a "synthetic hematocrit" derived from the native blood $\mathrm{T} 1$ values for the particular technique used. ${ }^{52}$

\section{REPORTING}

As with scanning protocols, reporting of CMR parametric mapping currently is not standardized but there are general consensus recommendations published by SCMR. ${ }^{8311477}$ The standardized image interpretation and post-processing in cardiovascular magnetic resonance 2020 update recommends at least the following when reporting mapping for clinical use $\mathrm{e}^{47}$ :

1) For clinical reports, the type of pulse sequence, reference range, and type/dose of gadolinium contrast agent (if applied) should be quoted.

2) Mapping results should include the numerical absolute value, the Z-score (number of standard deviations by which the result differs from the local normal mean; if available), and the normal range of the CMR system.

3) Local results should be benchmarked against published reported ranges, but a local reference range should be primarily used.

4) Reference ranges should be generated from data sets that were acquired, processed, and analyzed in the same way as the intended application, with the upper and lower range of normal defined by the mean $\pm 2 \mathrm{SD}$ of the normal data, respectively. 

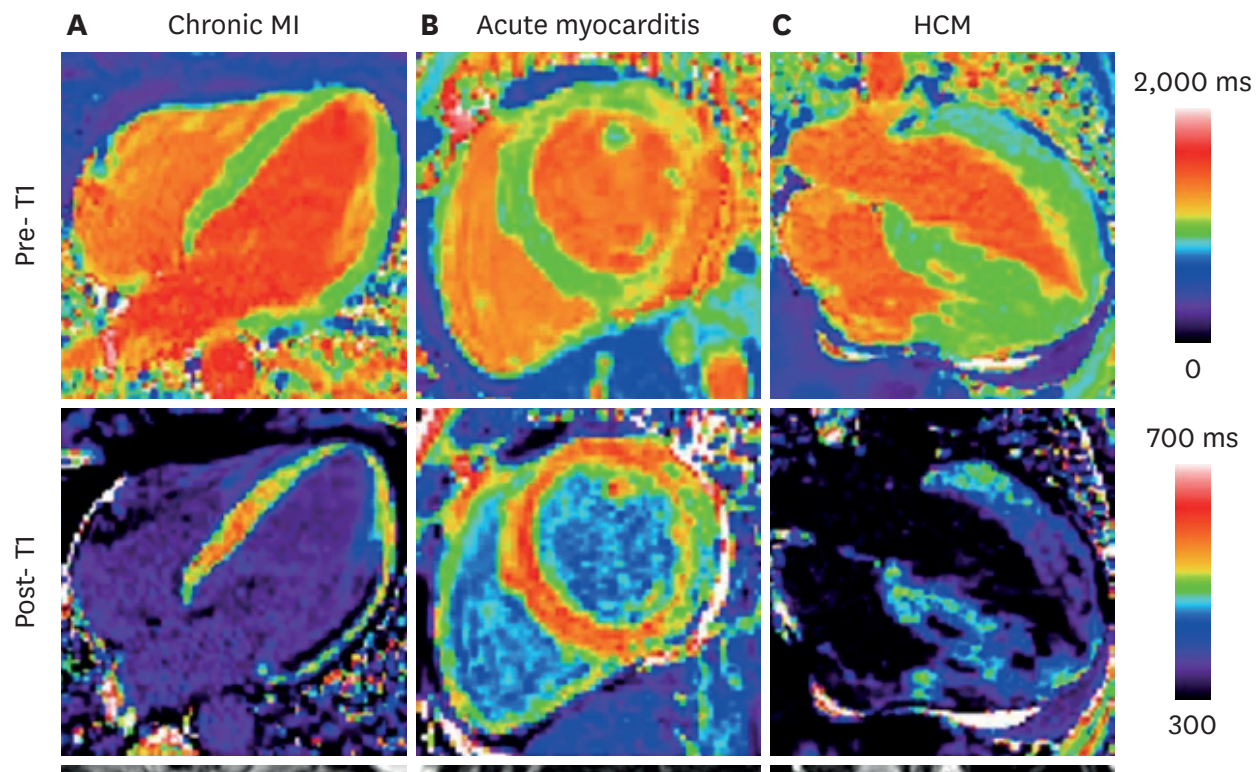

$700 \mathrm{~ms}$
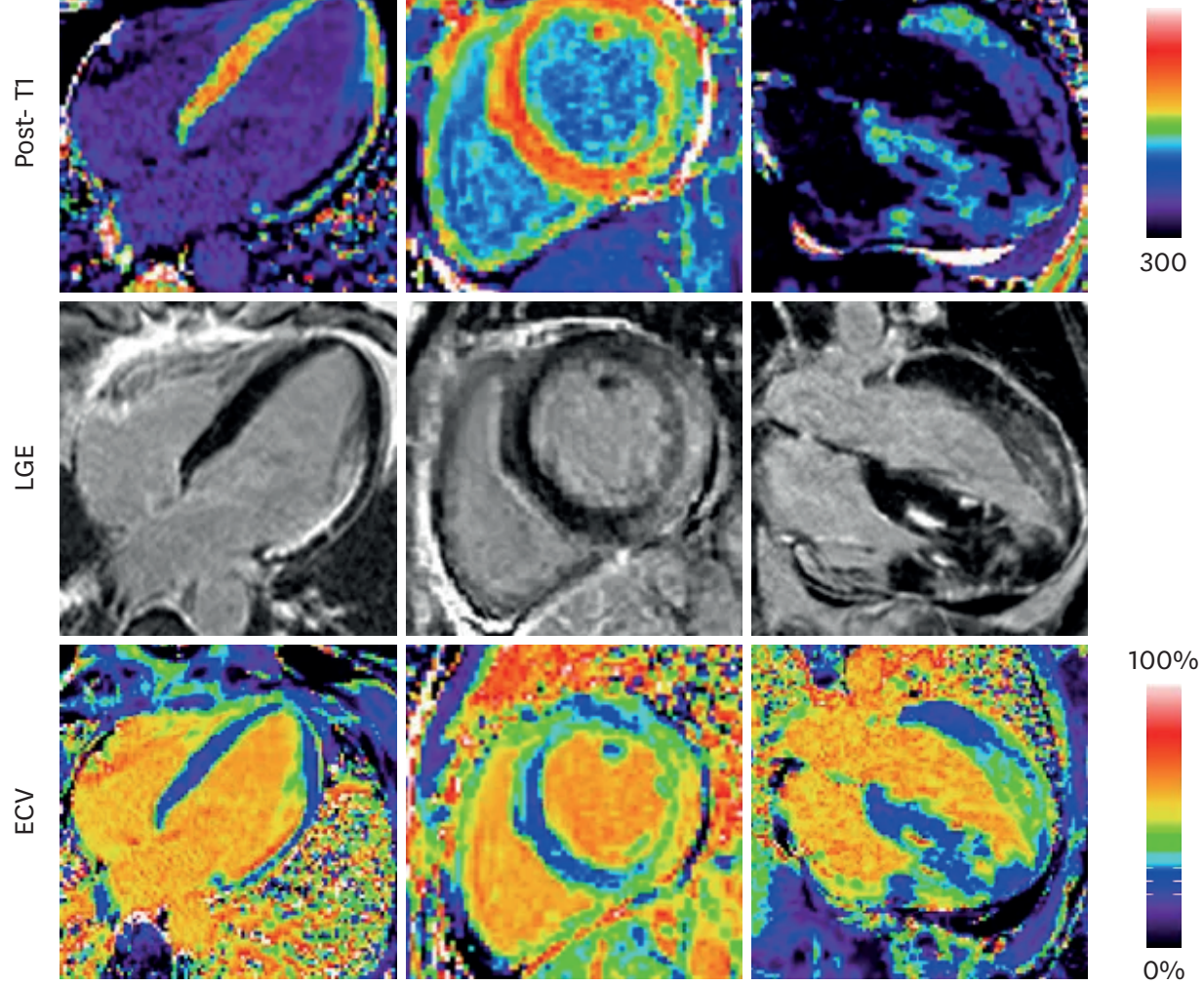

Figure 9. Examples illustrating excellent agreement between LGE and ECV in cases of focal abnormalities in myocardial ECV. Pre-contrast T1-maps (top row), post-contrast T1-maps (2nd row), late gadolinium enhancement (3rd row), and ECV maps (bottom row) for patients with: (A) chronic MI, (B) acute myocarditis, and (C) HCM. As originally published by BioMed Central in Kellman et al. Journal of Cardiovascular Magnetic Resonance 2012;14:64.51) $\mathrm{ECV}$ = extracellular volume; $\mathrm{HCM}$ = hypertrophic cardiomyopathy; $\mathrm{LGE}$ = late gadolinium enhancement; $\mathrm{MI}$ = myocardial infarction.

5) Parameter values should only be compared to other parameter values if they are obtained under similar conditions. In other words, the acquisition scheme, field strength, contrast agent and processing approach should be the same, and the results should be reported along with corresponding reference ranges for the given methodology.

In addition to the above, the authors also recommend including the following:

6) Recording the quality of $\mathrm{T} 1 / \mathrm{T} 2$ maps before using for clinical diagnosis, via assessment of T1/T2 curve-fits and/or quality controls maps (such as $\mathrm{R}^{2}$ or residual maps)

7) Report the number of slices obtained and their imaging plane prescription (e.g. 3 SA slices covering the LV)

8) Consistent and standardized training of image analysis and post-processing of parametric maps $^{48)}$ 
9) In additional to global T1/T2 values, the extent of any regional abnormalities based on segmental analysis, and the range of these values (e.g. "The basal-mid inferolateral segments have areas with significantly elevated T1 values, ranging from 1,000-1,050 ms")

10) An interpretation or differential diagnosis of the imaging findings within the clinical context of the referral (e.g. "...which may be consistent with acute myocardial inflammation in these areas")

\section{CHALLENGES AND FUTURE DIRECTION}

The clinical CMR community has a strong desire to implement parametric mapping for widespread clinical use, but the main challenge is the lack of standardization. Currently, there are many different T1- and T2-mapping techniques, each with their own metrological properties, normal ranges, sensitivity to disease and varying degrees of clinical validation and evidence-base. For an individual MRI centre that would like to implement parametric mapping, it is often confusing to know which T1- or T2-mapping method to use, and how to ensure that the method has been installed correctly before using it for systematic research or clinical applications. Additionally, the establishment of a local normal range is required according to current consensus, with each centre having to scan up to 50 normal volunteers. ${ }^{8}$ This is time-consuming and costly for individual MRI centres, especially clinical or private centres that are not focussed primarily on research. Establishing the local normal range needs to be repeated when there are major scanner upgrades or changes in the hardware or software platforms, which makes this prohibitive for many centres. Clearly, a better, more efficient and economical solution is required, such as the use of dedicated phantoms trialled since 2013 as part of the large, multicentre international Hypertrophic Cardiomyopathy Registry (HCMR). ${ }^{53}$

\section{CONCLUSION}

CMR parametric mapping is widely regarded as the 4th era of myocardial CMR after cine, LGE and perfusion imaging. The derived T1-, T2- and ECV relaxometries are in prime time for advanced quantitative myocardial tissue characterization. Myocardial T1 and T2 relaxation times correlate to histopathology in a range of myocardial diseases, and are especially useful in the evaluation of myocarditis or acute myocardial injury, suspected infiltration (amyloidosis, Anderson-Fabry disease, iron overload), and heart failure of unclear etiology. Parametric mapping techniques demonstrate narrow normal ranges and are very reproducible when the method is kept stable. Importantly, parametric mapping show sensitivity to a wide range of cardiac diseases, and may be used to track disease progression, response to therapy, and are increasingly included as endpoints for clinical trials. Parametric mapping adds incremental diagnostic and prognostic value to conventional tissue characterization techniques in the evaluation of myocardial diseases, and holds promise to offer contrast-free protocols in the future. Further progress hinges on standardization of acquisition methods, image post-processing and interpretation, which will allow widespread clinical use and the construction of large clinical databases to answer important clinical questions that smaller datasets cannot. CMR parametric mapping is expected to occupy an important role in the work-up of many cardiac diseases, optimizing clinical management pathways, and ultimately benefitting patients. 


\section{REFERENCES}

1. Čelutkienė J, Plymen CM, Flachskampf FA, et al. Innovative imaging methods in heart failure: a shifting paradigm in cardiac assessment. Position statement on behalf of the Heart Failure Association of the European Society of Cardiology. Eur J Heart Fail 2018;20:1615-33. PUBMED | CROSSREF

2. Ferreira VM, Schulz-Menger J, Holmvang G, et al. Cardiovascular magnetic resonance in nonischemic myocardial inflammation: expert recommendations. J Am Coll Cardiol 2018;72:3158-76. PUBMED | CROSSREF

3. Schulz-Menger J, Bluemke DA, Bremerich J, et al. Standardized image interpretation and post processing in cardiovascular magnetic resonance: Society for Cardiovascular Magnetic Resonance (SCMR) board of trustees task force on standardized post processing. J Cardiovasc Magn Reson 2013;15:35. PUBMED | CROSSREF

4. Ferreira VM, Piechnik SK, Dall'Armellina E, et al. Native T1-mapping detects the location, extent and patterns of acute myocarditis without the need for gadolinium contrast agents. J Cardiovasc Magn Reson 2014;16:36. PUBMED | CROSSREF

5. Formenti M. Mean of ratios or ratio of means: statistical uncertainty applied to estimate multiperiod probability of default. arXiv 2014:1409.4896.

6. Robson MD, Piechnik SK, Tunnicliffe EM, Neubauer S. T1 measurements in the human myocardium: the effects of magnetization transfer on the SASHA and MOLLI sequences. Magn Reson Med 2013;70:664-70. PUBMED | CROSSREF

7. Kellman P, Bandettini WP, Mancini C, Hammer-Hansen S, Hansen MS, Arai AE. Characterization of myocardial T1-mapping bias caused by intramyocardial fat in inversion recovery and saturation recovery techniques. J Cardiovasc Magn Reson 2015;17:33. PUBMED | CROSSREF

8. Messroghli DR, Moon JC, Ferreira VM, et al. Clinical recommendations for cardiovascular magnetic resonance mapping of T1, T2, T2^ and extracellular volume: a consensus statement by the Society for Cardiovascular Magnetic Resonance (SCMR) endorsed by the European Association for Cardiovascular Imaging (EACVI). J Cardiovasc Magn Reson 2017;19:75. PUBMED | CROSSREF

9. Messroghli DR, Radjenovic A, Kozerke S, Higgins DM, Sivananthan MU, Ridgway JP. Modified Look-Locker inversion recovery (MOLLI) for high-resolution T1 mapping of the heart. Magn Reson Med 2004;52:141-6. PUBMED | CROSSREF

10. Piechnik SK, Ferreira VM, Dall'Armellina E, et al. Shortened Modified Look-Locker Inversion recovery (ShMOLLI) for clinical myocardial T1-mapping at 1.5 and $3 \mathrm{~T}$ within a 9 heartbeat breathhold. J Cardiovasc Magn Reson 2010;12:69. PUBMED | CROSSREF

11. Piechnik SK, Ferreira VM, Lewandowski AJ, et al. Normal variation of magnetic resonance T1 relaxation times in the human population at 1.5 T using ShMOLLI. J Cardiovasc Magn Reson 2013;15:13. PUBMED | CROSSREF

12. Dabir D, Child N, Kalra A, et al. Reference values for healthy human myocardium using a T1 mapping methodology: results from the International T1 Multicenter cardiovascular magnetic resonance study. $J$ Cardiovasc Magn Reson 2014;16:69. PUBMED | CROSSREF

13. Liu JM, Liu A, Leal J, et al. Measurement of myocardial native T1 in cardiovascular diseases and norm in 1291 subjects. J Cardiovasc Magn Reson 2017;19:74. PUBMED | CROSSREF

14. Ferreira VM, Piechnik SK, Dall'Armellina E, et al. Non-contrast T1-mapping detects acute myocardial edema with high diagnostic accuracy: a comparison to T2-weighted cardiovascular magnetic resonance. $J$ Cardiovasc Magn Reson 2012;14:42. PUBMED | CROSSREF

15. Ferreira VM, Piechnik SK, Dall'Armellina E, et al. T(1) mapping for the diagnosis of acute myocarditis using CMR: comparison to T2-weighted and late gadolinium enhanced imaging. JACC Cardiovasc Imaging 2013;6:1048-58. PUBMED | CROSSREF

16. Dall'Armellina E, Piechnik SK, Ferreira VM, et al. Cardiovascular magnetic resonance by non contrast T1-mapping allows assessment of severity of injury in acute myocardial infarction. J Cardiovasc Magn Reson 2012;14:15. PUBMED | CROSSREF 
17. Karamitsos TD, Piechnik SK, Banypersad SM, et al. Noncontrast T1 mapping for the diagnosis of cardiac amyloidosis. JACC Cardiovasc Imaging 2013;6:488-97. PUBMED | CROSSREF

18. Sado DM, Maestrini V, Piechnik SK, et al. Noncontrast myocardial T1 mapping using cardiovascular magnetic resonance for iron overload. J Magn Reson Imaging 2015;41:1505-11. PUBMED | CROSSREF

19. Sado DM, White SK, Piechnik SK, et al. Identification and assessment of Anderson-Fabry disease by cardiovascular magnetic resonance noncontrast myocardial T1 mapping. Circ Cardiovasc Imaging 2013;6:392-8. PUBMED | CROSSREF

20. Feng $\mathrm{Y}, \mathrm{He} \mathrm{T}$, Carpenter JP, et al. In vivo comparison of myocardial $\mathrm{T} 1$ with $\mathrm{T} 2$ and $\mathrm{T} 2{ }^{\star}$ in thalassaemia major. J Magn Reson Imaging 2013;38:588-93.

PUBMED | CROSSREF

21. Thompson RB, Chow K, Khan A, et al. T1 mapping with cardiovascular MRI is highly sensitive for Fabry disease independent of hypertrophy and sex. Circ Cardiovasc Imaging 2013;6:637-45. CROSSREF

22. Dall'Armellina E, Ferreira VM, Kharbanda RK, et al. Diagnostic value of pre-contrast T1 mapping in acute and chronic myocardial infarction. JACC Cardiovasc Imaging 2013;6:739-42. PUBMED | CROSSREF

23. Liu D, Borlotti A, Viliani D, et al. CMR native T1 mapping allows differentiation of reversible versus irreversible myocardial damage in ST-segment-elevation myocardial infarction: an OxAMI Study (Oxford Acute Myocardial Infarction). Circ Cardiovasc Imaging 2017;10:e005986-005986. PUBMED | CROSSREF

24. Alkhalil M, Borlotti A, De Maria GL, et al. Hyper-acute cardiovascular magnetic resonance T1 mapping predicts infarct characteristics in patients with ST elevation myocardial infarction. J Cardiovasc Magn Reson 2020;22:3. PUBMED | CROSSREF

25. Wamil M, Borlotti A, Liu D, et al. Combined T1-mapping and tissue tracking analysis predicts severity of ischemic injury following acute STEMI-an Oxford Acute Myocardial Infarction (OxAMI) study. Int J Cardiovasc Imaging 2019;35:1297-308. PUBMED | CROSSREF

26. Banypersad SM, Fontana M, Maestrini V, et al. T1 mapping and survival in systemic light-chain amyloidosis. Eur Heart J 2015;36:244-51. PUBMED | CROSSREF

27. Fontana M, Banypersad SM, Treibel TA, et al. Native T1 mapping in transthyretin amyloidosis. JACC Cardiovasc Imaging 2014;7:157-65.

PUBMED | CROSSREF

28. Fontana M, White SK, Banypersad SM, et al. Comparison of T1 mapping techniques for ECV quantification. Histological validation and reproducibility of ShMOLLI versus multibreath-hold T1 quantification equilibrium contrast CMR. J Cardiovasc Magn Reson 2012;14:88. PUBMED | CROSSREF

29. Ferreira VM, Messroghli D. Mapping techniques. In: The EACVI Textbook of Cardiovascular Magnetic Resonance. Lombardi M, Plein S, Petersen S, Bucciarelli-Ducci C, Valsangiacomo Buechel ER, Basso C, Ferrari V, editors. Oxford, UK: Oxford University Press; 2018. p.133-45.

30. Dass S, Suttie JJ, Piechnik SK, et al. Myocardial tissue characterization using magnetic resonance noncontrast t1 mapping in hypertrophic and dilated cardiomyopathy. Circ Cardiovasc Imaging 2012;5:726-33. PUBMED | CROSSREF

31. Moon JC, Messroghli DR, Kellman P, et al. Myocardial T1 mapping and extracellular volume quantification: a Society for Cardiovascular Magnetic Resonance (SCMR) and CMR Working Group of the European Society of Cardiology consensus statement. J Cardiovasc Magn Reson 2013;15:92. PUBMED | CROSSREF

32. Flett AS, Hayward MP, Ashworth MT, et al. Equilibrium contrast cardiovascular magnetic resonance for the measurement of diffuse myocardial fibrosis: preliminary validation in humans. Circulation 2010;122:138-44. PUBMED | CROSSREF

33. White SK, Sado DM, Fontana M, et al. T1 mapping for myocardial extracellular volume measurement by CMR: bolus only versus primed infusion technique. JACC Cardiovasc Imaging 2013;6:955-62. PUBMED | CROSSREF 
34. Piechnik SK, Jerosch-Herold M. Myocardial T1 mapping and extracellular volume quantification: an overview of technical and biological confounders. Int J Cardiovasc Imaging 2018;34:3-14. PUBMED | CROSSREF

35. Liu A, Wijesurendra RS, Francis JM, et al. Adenosine stress and rest T1 mapping can differentiate between ischemic, infarcted, remote, and normal myocardium without the need for gadolinium contrast agents. JACC Cardiovasc Imaging 2016;9:27-36. PUBMED | CROSSREF

36. Mahmod M, Piechnik SK, Levelt E, et al. Adenosine stress native T1 mapping in severe aortic stenosis: evidence for a role of the intravascular compartment on myocardial T1 values. J Cardiovasc Magn Reson 2014;16:92. PUBMED | CROSSREF

37. Wong TC, Piehler K, Meier CG, et al. Association between extracellular matrix expansion quantified by cardiovascular magnetic resonance and short-term mortality. Circulation 2012;126:1206-16. PUBMED | CROSSREF

38. Wong TC, Piehler KM, Kang IA, et al. Myocardial extracellular volume fraction quantified by cardiovascular magnetic resonance is increased in diabetes and associated with mortality and incident heart failure admission. Eur Heart J 2014;35:657-64. PUBMED | CROSSREF

39. Piechnik SK, Neubauer S, Ferreira VM. State-of-the-art review: stress T1 mapping-technical considerations, pitfalls and emerging clinical applications. MAGMA 2018;31:131-41. PUBMED | CROSSREF

40. Nakamori S, Fahmy A, Jang J, et al. Changes in myocardial native $\mathrm{T}_{1}$ and $\mathrm{T}_{2}$ after exercise stress: a noncontrast CMR pilot study. JACC Cardiovasc Imaging 2020;13:667-80. PUBMED | CROSSREF

41. Giri S, Chung YC, Merchant A, et al. T2 quantification for improved detection of myocardial edema. $J$ Cardiovasc Magn Reson 2009;11:56. PUBMED | CROSSREF

42. Sprinkart AM, Luetkens JA, Träber F, et al. Gradient spin echo (GraSE) imaging for fast myocardial T2 mapping. J Cardiovasc Magn Reson 2015;17:12.

PUBMED | CROSSREF

43. Fernández-Jiménez R, Sánchez-González J, Aguero J, et al. Fast T2 gradient-spin-echo (T2-GraSE) mapping for myocardial edema quantification: first in vivo validation in a porcine model of ischemia/ reperfusion. J Cardiovasc Magn Reson 2015;17:92. PUBMED | CROSSREF

44. Abdel-Aty H, Boyé P, Zagrosek A, et al. Diagnostic performance of cardiovascular magnetic resonance in patients with suspected acute myocarditis: comparison of different approaches. J Am Coll Cardiol 2005;45:1815-22. PUBMED | CROSSREF

45. Kramer CM, Barkhausen J, Bucciarelli-Ducci C, Flamm SD, Kim RJ, Nagel E. Standardized cardiovascular magnetic resonance imaging (CMR) protocols: 2020 update. J Cardiovasc Magn Reson 2020;22:17. PUBMED | CROSSREF

46. von Knobelsdorff-Brenkenhoff F, Prothmann M, Dieringer MA, et al. Myocardial T1 and T2 mapping at 3 T: reference values, influencing factors and implications. J Cardiovasc Magn Reson 2013;15:53. PUBMED | CROSSREF

47. Schulz-Menger J, Bluemke DA, Bremerich J, et al. Standardized image interpretation and post-processing in cardiovascular magnetic resonance - 2020 update: Society for Cardiovascular Magnetic Resonance (SCMR): Board of Trustees Task Force on Standardized Post-Processing. J Cardiovasc Magn Reson 2020;22:19. PUBMED | CROSSREF

48. Carapella V, Puchta H, Lukaschuk E, et al. Standardized image post-processing of cardiovascular magnetic resonance T1-mapping reduces variability and improves accuracy and consistency in myocardial tissue characterization. Int J Cardiol 2020;298:128-34. PUBMED | CROSSREF

49. Baeßler B, Schaarschmidt F, Dick A, et al. Mapping tissue inhomogeneity in acute myocarditis: a novel analytical approach to quantitative myocardial edema imaging by T2-mapping. J Cardiovasc Magn Reson 2015;17:115. PUBMED | CROSSREF

50. Meduri A, Di Molfetta DV, Natale L, Manfredi R. Cardiac magnetic resonance in systemic sclerosis patients with cardiac symptoms. Eur Rev Med Pharmacol Sci 2017;21:4797-803. PUBMED 
51. Kellman P, Wilson JR, Xue H, et al. Extracellular volume fraction mapping in the myocardium, part 2: initial clinical experience. J Cardiovasc Magn Reson 2012;14:64.

PUBMED | CROSSREF

52. Treibel TA, Fontana M, Maestrini V, et al. Automatic measurement of the myocardial interstitium: synthetic extracellular volume quantification without hematocrit sampling. JACC Cardiovasc Imaging 2016;9:54-63.

PUBMED | CROSSREF

53. Kramer CM, Appelbaum E, Desai MY, et al. Hypertrophic Cardiomyopathy Registry: the rationale and design of an international, observational study of hypertrophic cardiomyopathy. Am Heart J 2015;170:223-30. PUBMED | CROSSREF 